\title{
CONJUNCTIVAL POCKETS*
}

BY

\author{
S. AYOUB \\ Cairo
}

SYMBLEPHARON is described by all authors discussing conjunctival diseases as adhesion between the palpebral and bulbar conjunctivae. Its aetiology is described as purulent conjunctivitis, diphtheritic conjunctivitis, trachoma, trauma, and so on. These factors do not always cause symblepharon, but may result in a very troublesome condition, apparently not mentioned in the literature, viz. conjunctival pocketing.

Two similar cases have been seen in one year, in which the patient complained of recurrent mucopurulent conjunctivitis in one eye which was not very severe and responded to the usual treatment for conjunctivitis only after prolonged application. Between attacks the patient felt some discomfort in the affected eye.

\section{Case Report}

The attacks started in a 2-year-old boy after a severe purulent conjunctivitis, and continued several times a year up to the age of 21 years.

Cultures from the conjunctiva at various times showed various organisms and the attacks recurred even though on many occasions the cultures were sterile.

When the upper eyelid was everted (Figure), raised bands were seen on the palpebral


conjunctival surface and in some areas pouches had formed behind them. It was thought that these pouches collected bacteria and mucus, and that this started the inflammation. Even without bacterial growth the rough surface might irritate the conjunctiva and cause discomfort.

A plastic operation to smooth the surface by making conjunctival flaps and removing the fibrous bands or by grafting mucous membrane from the ch $\mathrm{kk}$ was advised, but operation was refused and the disease

FIGURE.-Conjunctival surface of upper lid. continued.

An extensive search of the literature revealed only a remark made by Atkinson (1934): "in as much as with movements of the eye traction is made at the site of adhesion the eye gets into an irritated condition". No mention was found of the collection of mucus in conjunctival pockets.

\section{REFERENCE}

AtKinson, D. T. (1934). " "External Diseases of the Eye". Kimpton, London; Lea and Febiger, Philadelphia.

* Received for publication May 11, 1962. 